\title{
Influence of cataract surgery on macular vascular density in patients with myopia using optical coherence tomography angiography
}

\author{
TINGTING LI ${ }^{1}$, AWOKE GUADIE ${ }^{2}$, LE FENG $^{1}$, JIANWU FAN ${ }^{1}$, ZIYUAN JIANG ${ }^{3}$ and FANG LIU ${ }^{1}$ \\ ${ }^{1}$ Department of Ophthalmology, 10th People's Hospital Affiliated to Tongji University, Shanghai 200072, \\ P.R. China; ${ }^{2}$ Department of Biology, College of Natural Sciences, Arba Minch University, \\ 21 Arba Minch, Ethiopia; ${ }^{3}$ Department of Ophthalmology, Clinical Medical College of \\ Shanghai 10th People's Hospital of Nanjing Medical University, Shanghai 200072, P.R. China
}

Received March 6, 2020; Accepted July 1, 2020

DOI: $10.3892 /$ etm.2020.9388

\begin{abstract}
The aim of the present study was to evaluate macular vascular density (MVD) variation after uneventful cataract surgery using optical coherence tomography angiography (OCTA) in patients with high myopia. Patients with cataracts scheduled for cataract surgery were divided into a high-myopia group [spherical equivalent (SE) $\leq-6.0$ diopter (D) and axial length $(\mathrm{AL}) \geq 25 \mathrm{~mm}$ ] and a low-myopia group (SE>-6.0 D and $\mathrm{AL}<25 \mathrm{~mm}$ ). All patients were examined for MVD and retinal thickness (RT) with OCTA pre-operatively and post-operatively ( 1 day, 1 week, and 1 and 3 months after surgery). A total of 55 eyes from 44 patients were included. MVD and RT both changed after cataract surgery. The mean change in superficial vascular density (SVD) in patients with high myopia was significantly lower than that in the low-myopia group at the four post-operative time-points (all $\mathrm{P}<0.05$ ). In addition, the RT of eyes with high myopia exhibited a different variation compared with that of the low-myopia group. Significant correlations were identified between AL, RT, intraocular pressure and SVD after surgery. In conclusion, superficial retinal perfusion in patients with high myopia was significantly lower than that in low myopia patients, which may lead to complications caused by poor perfusion.
\end{abstract}

\section{Introduction}

Age-associated cataracts are the most common disease that causes blindness worldwide (1). The gradual decline in

Correspondence to: Professor Fang Liu, Department of Ophthalmology, 10th People's Hospital Affiliated to Tongji University, 301 Yanchangzhong Road, Shanghai 200072, P.R. China E-mail: fangliu_2004@yahoo.com

Key words: cataract, high myopia, low myopia, phacoemulsification, optical coherence tomography angiography, macular vascular density visual acuity (VA) affects the quality of life of the elderly. Phacoemulsification combined with intraocular lens implantation is an effective treatment method to improve VA (2). However, certain patients with cataracts experience a decrease in VA at different time-points after surgery $(3,4)$. For instance, Emeriewen et al (3) studied the decreased VA after cataract surgery caused by ischemic optic neuropathy and reported that hypertension, diabetes, cerebrovascular disease and high levels of lipids were systemic risk factors. It was reported that the best-corrected visual acuity (BCVA) was positively correlated with macular flow density in pathological myopia, and that the macular vascular system was essential for visual function (5). With the extension of the ocular axis, certain structural changes occur in high myopia, including retinal and choroidal thickness thinning, optic disc atrophy and reduction of the retinal nerve fiber layer (RNFL) thickness (6). Fan et al (7) also indicated that macular vascular density (MVD) and ganglion cell complex thickness, which consists of the inner plexiform layer (IPL), ganglion cell layer (GCL) and nerve fiber layer, decreased with increasing myopia. In addition, Benavente-Pérez et al (8) indicated that both retinal blood flow and blood vessel diameter decreased in high myopia based on Doppler ultrasound. However, whether uneventful cataract surgery is able to affect the perfusion of the macular area in high myopia has remained elusive.

Retinal blood flow may be observed by a variety of methods, including Doppler ultrasound and fundus fluorescein angiography (FFA). However, there are certain limitations to these clinical applications, since Doppler ultrasound may only visualize large vessels with low resolution (9), while FFA is invasive and difficult to quantify (5). Optical coherence tomography angiography (OCTA) with split-spectrum amplitude-decorrelation angiography (SSADA) is able to generate blood flow images of the retinal and choroidal vessels without contrast agents. This technique is non-invasive, has no complications or adverse reactions and provides a high-definition, three-dimensional image (10). More importantly, this technique is able to provide morphological information about retinal vessels and quantify vascular density (11). 
In the present study, OCTA was used to measure the MVD of cataracts and to assess the effects of uneventful cataract surgery on retinal perfusion in high myopia.

\section{Materials and methods}

Patients. The present study was a prospective observational study. Patients who were scheduled for cataract surgery at the Department of Ophthalmology of the 10th People's Hospital affiliated to Tongji University (Shanghai) were enrolled between May 2016 and March 2017. Outpatients with complaints of painless and gradual blurry vision in addition to lens opacity were diagnosed with cataracts after slit-lamp examination. Lens opacification was scored after pupil dilation with the compound tropicamide using the Lens Opacification Classification System III (12). The nuclear opalescence (NO), nuclear color (NC), cortical cataract (C) and posterior subcapsular cataract $(\mathrm{P})$ were used as references to classify lens opacities under the slit lamp. Patients with cataracts who were aged $>50$ years and scored $\geq N O 3-N C 3$ or $\geq \mathrm{C} 3$ or $\geq \mathrm{P} 3$ were included. The exclusion criteria comprised glaucoma, cornea diseases, retinal vascular diseases, previous intraocular surgery, trauma and other pre-existing ocular diseases affecting vision. Furthermore, patients with systematic diseases, including hypertension, diabetes, systemic cardiovascular diseases and hematological diseases were also excluded. All participants were divided into a high-myopia group [group A; spherical equivalent $(\mathrm{SE}) \leq-6.0 \mathrm{D}$ and axis length $(\mathrm{AL}) \geq 25 \mathrm{~mm}]$ or a low-myopia group (group B; SE $>-6.0 \mathrm{D}$ and $\mathrm{AL}<25 \mathrm{~mm}$ ). Informed consent was obtained from all the participants and the study protocol was approved by the Clinical Research Ethical Committee of Shanghai 10th People's Hospital affiliated to Tongji University (Shanghai, China).

Examinations. All patients underwent routine examinations prior to cataract surgery, including best-corrected visual acuity (BCVA), intraocular pressure (IOP), refraction, corneal endothelial cell counting, corneal topography, examination with Intraocular Lenses Master (Carl Zeiss AG), fundus photography, OCTA and ophthalmoscopic examination with slit lamp. After the pre-operative dilation of the pupil with tropicamide, surgery was performed by the same skilled physician using Infinity (Alcon Laboratories). All patients were examined for MVD and retinal thickness (RT) with OCTA on the first day, and at 1 week, 1 and 3 months after surgery. All patients with high myopia were classified according to the classification of Curtin (13), including type I (posterior staphyloma), type II (macular staphyloma), type III (peripapillary staphyloma), type VI (combined staphyloma, types I and II) and type VII (combined staphyloma, types I and III).

All operations were performed by the same surgeon(FL) and the irrigation pressure (IP) was maintained at $80-85 \mathrm{~cm} \mathrm{H}_{2} \mathrm{O}$ during surgery. All participants underwent measurement of superficial vascular density (SVD) and deep vascular density (DVD) by a skilled technician prior to and after cataract surgery (1 day, 1 week, 1 and 3 months post-operatively). Fundus vascular scanning was performed with an RTVue-XR Avanti system (Optovue Inc.) with a wavelength of $840 \mathrm{~nm}$ (bandwidth of $50 \mathrm{~nm}$ ) and an en-face OCTA scan of $3 \times 3 \mathrm{~mm}$ over the macula. The subjects were requested to stare at the blue sighting mark with blinking for at least $3 \mathrm{sec}$ and the retinal stratified blood flow images were obtained by horizontal and vertical scans. Both the SVD [3 $\mu \mathrm{m}$ beneath the inner limiting membrane (ILM) to $16 \mu \mathrm{m}$ beneath the IPL] and DVD (16 $\mu \mathrm{m}$ beneath the IPL to $71 \mu \mathrm{m}$ beneath the IPL) were quantified (Fig. 1A and B). The segments included the fovea (1 mm circular area centered on the fovea) and parafovea (annulus with an outer diameter of $3 \mathrm{~mm}$ and an inner diameter of $1 \mathrm{~mm}$ centered on the fovea). The parafovea was divided into the superior-hemi ( $\mathrm{SH})$, inferior-hemi (IH), temporal (T), superior (S), nasal (N) and inferior (I) quadrants. The full RT (from the ILM to the retinal pigment epithelium) and the superficial RT (from the ILM to the IPL) were also evaluated. MVD and RT were automatically calculated using the built-in software (AngioAnalytics) of the RTVue XR-Avanti system. Poor-quality images with a signal strength index of $<40$ and severe motion artifacts were excluded from the analysis.

Statistical analysis. Statistical analysis was performed with SPSS version 19.0 (IBM Corp.). Data were analyzed with the Kolmogorov-Smirnov test to evaluate their normal distribution. Pearson's $\chi^{2}$ test was performed to analyze gender differences. The data from pre-operative and post-operative measurements were analyzed by generalized estimating equations (14) with Bonferroni corrections. Unpaired t-test was used to analyze age, SE, AL, BCVA and IOP between the two groups. Pearson's correlation was used to further analyze the correlation between age, AL, RT, BCVA and MVD. P<0.05 was considered to indicate a statistically significant difference.

\section{Results}

Demographic and ocular characteristics of patients. A total of 57 eyes from 46 patients were included in the present study. The surgeries of all subjects were successfully completed with no complications during the operation. In total, 2 patients ( 2 eyes) were excluded due to macular edema at 1 month following the operation. Eventually, 55 eyes from 44 patients were included in the study (16 males and 28 females, including 12 patients with the two eyes examined), who had a mean age of $66.37 \pm 5.47$ years. There were 7 patients between 50 and 60 years of age, 27 patients between 60 to 70 years of age and 10 patients older than 70 years. A total of 24 eyes from 21 patients were included in group A, with a mean refraction of $-10.80 \pm 5.04 \mathrm{D}$ and an $\mathrm{AL}$ of $27.70 \pm 1.62 \mathrm{~mm}$. In total, 31 eyes of 23 patients were in group $\mathrm{B}$, with a mean refraction of $-0.76 \pm 2.21 \mathrm{D}$ and an AL of $23.22 \pm 0.95 \mathrm{~mm}$. There was no significant difference in sex $(\mathrm{P}=0.745)$ or age $(\mathrm{P}=0.08)$ between the two groups (Table I). In group A, 20 eyes were classified as type III, 2 eyes as type I and 1 eye each as types VI and VII (Table II). The mean pre-operative value for BCVA (logMAR) was $0.51 \pm 0.41$ for group $\mathrm{A}$ and $0.23 \pm 0.22$ for group $\mathrm{B}$. The BCVA of groups $\mathrm{A}$ and $\mathrm{B}$ were determined to be $0.03 \pm 0.05$ and $0.04 \pm 0.06$ at 3 months after surgery, respectively. The IOP of group A was $16.56 \pm 3.79,14.85 \pm 2.42,13.17 \pm 0.42$ and $13.38 \pm 1.86 \mathrm{mmHg}$ at 1 day, 1 week, 1 month and 3 months post-operatively, respectively. The IOP of group B was $16.36 \pm 3.16,12.71 \pm 2.15$, $11.54 \pm 0.24$ and $12.39 \pm 1.60 \mathrm{mmHg}$, respectively. Moreover, the IOP of high-myopia patients was significantly higher than that of low-myopia patients at 1 week $(\mathrm{P}=0.001), 1$ month $(\mathrm{P}=0.001)$ and 3 months $(\mathrm{P}=0.034)$ after surgery (Table $\mathrm{SI})$. 
Table I. Demographic and ocular characteristics of patients in the two groups.

\begin{tabular}{lccr}
\hline Characteristic & High myopia $(\mathrm{n}=24)$ & Low myopia $(\mathrm{n}=31)$ & P-value \\
\hline Age (years) & $65.32 \pm 6.01$ & $67.66 \pm 4.59$ & $0.080^{\mathrm{a}}$ \\
Gender (male/female) & $9 / 15$ & $11 / 20$ & $0.740^{\mathrm{b}}$ \\
SE (diopters) & $-10.80 \pm 5.04$ & $-0.76 \pm 2.21$ & $0.001^{\mathrm{a}}$ \\
Axial length (mm) & $27.70 \pm 1.62$ & $23.22 \pm 0.95$ & $0.001^{\mathrm{a}}$ \\
BCVA (logMAR) & $0.51 \pm 0.41$ & $0.23 \pm 0.22$ & $0.002^{\mathrm{a}}$ \\
IOP (mmHg) & $15.19 \pm 2.61$ & $14.63 \pm 2.67$ & $0.438^{\mathrm{a}}$ \\
\hline
\end{tabular}

${ }^{\mathrm{a}}$ Unpaired t-test; ${ }^{\mathrm{b}} \chi^{2}$ test. Values are expressed as the mean \pm standard deviation or $\mathrm{n}$. SE, spherical equivalent; BCVA, best-corrected visual acuity; IOP, intraocular pressure.
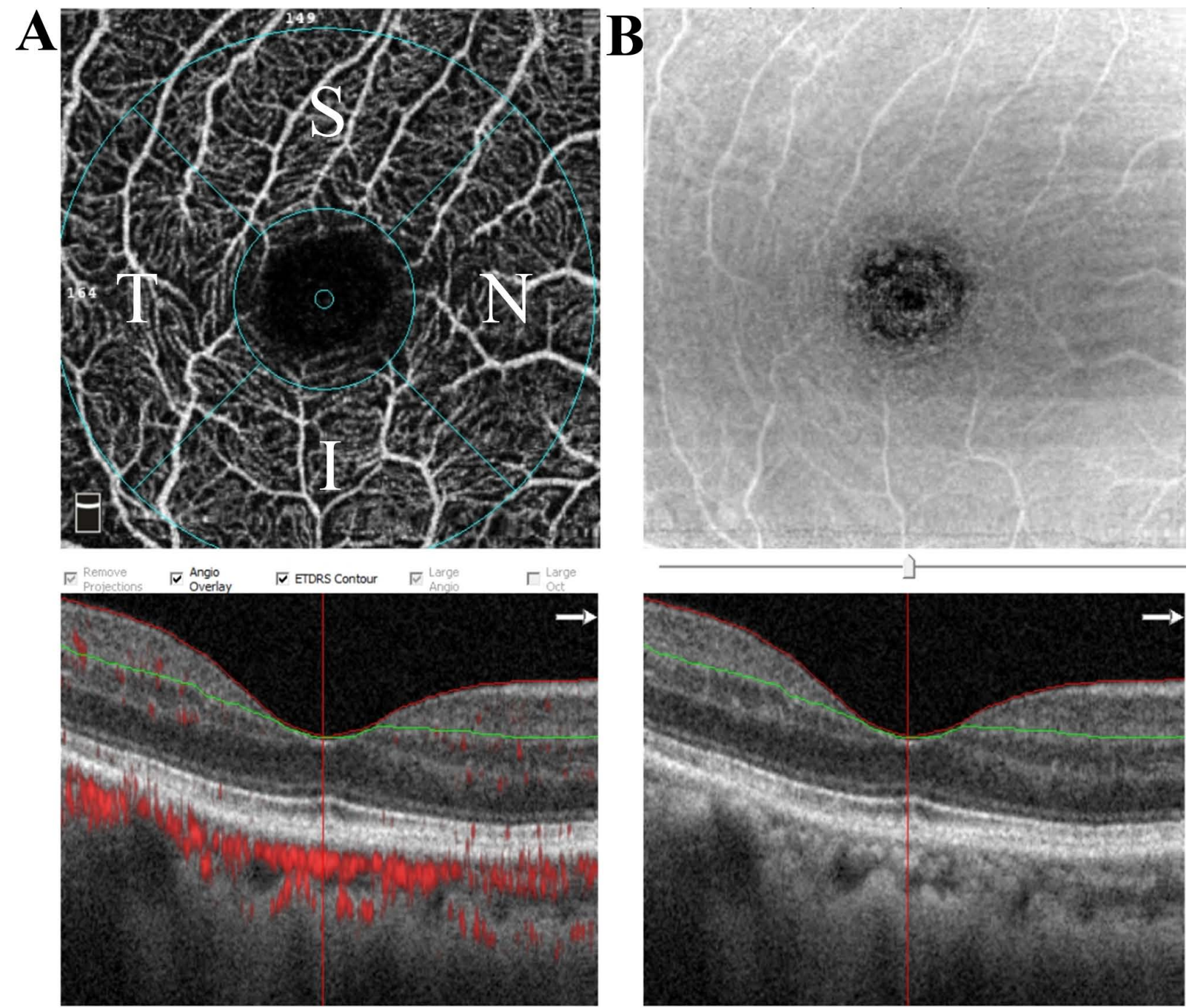

Figure 1. Determination of retinal vessels by optical coherence tomography angiography. (A) The horizontal (upper panel) and vertical (lower panel) scan of SVP. (B) The horizontal (upper panel) and vertical (lower panel) image of DVP. The parafovea of macular was divided into T, S, N, I quadrants. The green line indicates the line between SVP and DVP. The white arrow indicates the scan direction. SVP, superficial vascular plexus; DVP, deep vascular plexus; $\mathrm{T}$, temporal; S, superior; $\mathrm{N}$, nasal; I, inferior.

$M V D$ and $R T$ and their variations after cataract surgery. The generalized estimating equations suggested that the SVD and DVD of the parafovea changed in group A (Wald $\chi^{2}=13.65$, $\mathrm{P}=0.008$ and Wald $\chi^{2}=37.12, \mathrm{P}<0.001$, respectively) and group B (Wald $\chi^{2}=58.82, \mathrm{P}<0.001$ and Wald $\chi^{2}=19.71$,
$\mathrm{P}=0.001$, respectively), with an increasing trend within a month and gradually decreasing at 3 months after cataract surgery (Table III). Of note, in group A, there was a significant decrease at 3 months in both SVD and DVD [48.59 \pm 0.82 vs. $45.79 \pm 0.65(\mathrm{P}=0.005)$ and $57.69 \pm 0.65$ vs. $51.46 \pm 1.25$ 
Table II. The number and axis length in patients with different types of high myopia.

\begin{tabular}{llcc}
\hline Type & \multicolumn{1}{c}{ Description } & $\mathrm{n}(\%)$ & Axis length (mm) \\
\hline I & Posterior staphyloma & $2(8)$ & $29.56 \pm 0.07$ \\
II & Macular staphyloma & 0 & $/$ \\
III & Peripapillary staphyloma & $20(83)$ & $27.31 \pm 1.44$ \\
IV & Nasal staphyloma & 0 & $/$ \\
V & Inferior staphyloma & 0 & $/$ \\
VI & Combined staphyloma, Types I and II & $1(4)$ & 30.51 \\
VII & Combined staphyloma, types I and III & $1(4)$ & 29.87 \\
\hline
\end{tabular}

Table III. Macular vascular density and retinal thickness of two groups at baseline and 4 post-operative time-points.

A, High myopia $(n=24)$

\begin{tabular}{|c|c|c|c|c|c|c|c|}
\hline Parameters & Baseline & 1 day post-op & 1 week post-op & 1 month post-op & 3 months post-op & Wald $\chi^{2}$ & P-value \\
\hline $\mathrm{RT}$ in fovea (mm) & $243.43 \pm 4.86$ & $241.58 \pm 5.34$ & $242.66 \pm 5.03$ & $250.08 \pm 5.08$ & $242.78 \pm 3.83$ & 15.31 & 0.004 \\
\hline $\mathrm{RT}$ in parafovea $(\mathrm{mm})$ & $298.17 \pm 3.35$ & $293.00 \pm 3.59$ & $296.57 \pm 3.74$ & $304.79 \pm 3.31$ & $300.29 \pm 2.85$ & 50.22 & $<0.001$ \\
\hline $\operatorname{SVD}(\%)$ & $46.22 \pm 0.98$ & $46.34 \pm 1.01$ & $48.29 \pm 1.13$ & $48.59 \pm 0.82$ & $45.79 \pm 0.65$ & 13.65 & 0.008 \\
\hline $\operatorname{DVD}(\%)$ & $51.93 \pm 1.21$ & $54.56 \pm 1.24$ & $55.62 \pm 1.15$ & $57.69 \pm 0.65$ & $51.46 \pm 1.25$ & 37.12 & $<0.001$ \\
\hline
\end{tabular}

B, Low myopia $(n=31)$

\begin{tabular}{|c|c|c|c|c|c|c|c|}
\hline Parameters & Baseline & 1 day post-op & 1 week post-op & 1 month post-op & 3 months post-op & Wald $\chi^{2}$ & P-value \\
\hline RT in fovea (mm) & $248.75 \pm 5.61$ & $248.81 \pm 5.41$ & $249.52 \pm 5.04$ & $261.31 \pm 6.06$ & $263.55 \pm 2.02$ & 13.08 & 0.011 \\
\hline RT in parafovea (mm) & $313.60 \pm 3.54$ & $314.47 \pm 3.61$ & $315.47 \pm 3.33$ & $324.92 \pm 3.29$ & $326.93 \pm 1.75$ & 31.54 & $<0.001$ \\
\hline SVD (\%) & $46.30 \pm 0.73$ & $50.33 \pm 0.78$ & $51.84 \pm 0.72$ & $52.92 \pm 0.62$ & $50.91 \pm 0.68$ & 58.82 & $<0.001$ \\
\hline DVD $(\%)$ & $54.14 \pm 1.07$ & $57.31 \pm 0.60$ & $57.67 \pm 1.04$ & $59.22 \pm 0.71$ & $57.61 \pm 0.84$ & 19.71 & 0.001 \\
\hline
\end{tabular}

Comparison of data in two groups by generalized estimating equations. Values are expressed as the mean \pm standard deviation. RT, retinal thickness; SVD, superficial vascular density; DVD, deep vascular density.

$(\mathrm{P}<0.001)$, respectively] compared with the measurements at 1 month after surgery, which was even slightly lower than that at the baseline $(45.79 \pm 0.65$ vs. $46.22 \pm 0.98 ; \mathrm{P}>0.05)$. In group B, although the SVD and DVD decreased at 3 months after surgery, they were still significantly higher than the baseline values $(\mathrm{P}=0.001$ and $\mathrm{P}=0.008$, respectively; Fig. $2 \mathrm{~A})$. In the parafovea, the vascular density in different quadrants was consistent with the mean SVD in group A after surgery, including $\mathrm{SH}$ (Wald $\chi^{2}=22.52, \mathrm{P}<0.001$ ), IH (Wald $\chi^{2}=33.85$, $\mathrm{P}<0.001$ ), $\mathrm{T}$ (Wald $\chi^{2}=34.19, \mathrm{P}<0.001$ ), $\mathrm{S}$ (Wald $\chi^{2}=15.62$, $\mathrm{P}=0.004), \mathrm{N}$ (Wald $\chi^{2}=53.02, \mathrm{P}<0.001$ ) and I (Wald $\chi^{2}=16.75$, $\mathrm{P}=0.002)$. In group $\mathrm{B}$, there was also a significant change in the different quadrants after surgery (all $\mathrm{P}<0.05$; Table SII).

The whole RT of the fovea and parafovea also exhibited a different variation tendency over the four time-points after surgery between group A (Wald $\chi^{2}=15.31, \mathrm{P}=0.004$ and Wald $\chi^{2}=50.22, \mathrm{P}<0.001$ ) and group $\mathrm{B}$ (Wald $\chi^{2}=13.08, \mathrm{P}=0.011$ and Wald $\chi^{2}=31.54, \mathrm{P}<0.001$; Table III). In group A, the RT decreased on the first post-operative day and then gradually increased within a month and decreased again at 3 months after surgery. However, the RT in group B increased constantly over the 3 months after surgery and had a smaller volatility than that of group A (Fig. 2B). In group A, the RT in the parafovea was significantly changed after surgery in SH (Wald $\chi^{2}=12.75$, $\mathrm{P}=0.013$ ), $\mathrm{S}$ (Wald $\chi^{2}=11.21, \mathrm{P}=0.024$ ) and $\mathrm{N}$ (Wald $\chi^{2}=13.79$, $\mathrm{P}=0.008$ ), but not for IH (Wald $\chi^{2}=7.55, \mathrm{P}=0.11$ ) $\mathrm{T}$ (Wald $\chi^{2}=7.84, \mathrm{P}=0.098$ ) or I (Wald $\chi^{2}=6.43, \mathrm{P}=0.169$ ). However, in group $\mathrm{B}$, there was a significant difference in these quadrants (all $\mathrm{P}<0.05)$.

Changes in MVD within four post-operative time-points in the two groups. As presented in Table III, both the SVD and DVD of the two groups increased after surgery compared with those at the baseline. However, the SVD of group A exhibited a slight increase at 1 day, 1 week and 1 month post-operatively $(0.23,4.43$ and $5.06 \%$, respectively) and a slight decrease at 3 months (-1.21\%; all $\mathrm{P}>0.05)$, whereas a significant increase was obtained in group B at the four post-operative time-points (8.87, 12.15, 13.93 and 9.87\%, respectively; all $\mathrm{P}<0.05)$. In addition, the mean changes in SVD were significantly different 
Table IV. Mean changes of macular vascular density $(\%)$ in parafovea in high myopia and low myopia groups.

\begin{tabular}{|c|c|c|c|c|c|c|c|c|}
\hline \multirow[b]{2}{*}{ Time-point } & \multicolumn{4}{|c|}{ Superficial vascular density } & \multicolumn{4}{|c|}{ Deep vascular density } \\
\hline & High myopia & Low myopia & Wald $\chi^{2}$ & P-value & High myopia & Low myopia & Wald $\chi^{2}$ & P-value \\
\hline 1 Day post-op & $0.11 \pm 1.36$ & $4.11 \pm 0.69$ & 6.861 & 0.009 & $3.63 \pm 1.45$ & $3.21 \pm 0.90$ & 0.059 & 0.808 \\
\hline 1 Week post-op & $2.05 \pm 1.23$ & $5.63 \pm 0.91$ & 5.428 & 0.020 & $3.68 \pm 1.64$ & $3.58 \pm 1.34$ & 0.002 & 0.962 \\
\hline 1 Month post-op & $2.34 \pm 1.35$ & $6.45 \pm 0.91$ & 6.349 & 0.012 & $5.76 \pm 1.39$ & $5.03 \pm 1.22$ & 0.153 & 0.695 \\
\hline 3 Months post-op & $-0.52 \pm 1.11$ & $4.57 \pm 1.02$ & 11.353 & 0.001 & $0.53 \pm 1.35$ & $3.51 \pm 1.01$ & 3.077 & 0.079 \\
\hline
\end{tabular}

Comparison of changes in superficial vascular density and deep vascular density between two groups by generalized estimating equations after surgery. Values are expressed as the mean \pm standard deviation.
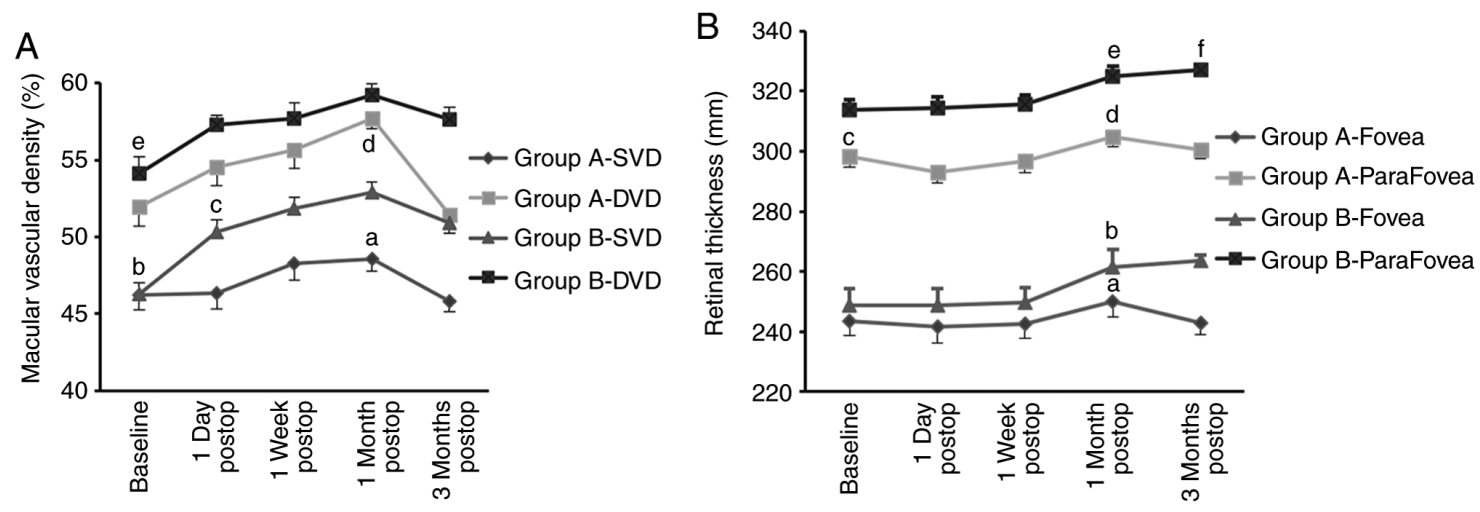

Figure 2. Variation in macular vascular density (expressed as a percentage) and RT (mm) of groups A and B prior to and after surgery. (A) Macular vascular density. $\mathrm{P}<0.05$ was determined for the following comparisons: A, 1 month post-op vs. 3 months post-op; $b$ and e, baseline vs. 1 day post-op, 1 week post-op, 1 month post-op and 3 months post-op; c, 1 day post-op vs. 1 month post-op; d, baseline vs. 1 month post-op. (B) RT in fovea and parafovea. P $<0.05$ was determined for the following comparisons: a, 1 month post-op vs. baseline and 1 week post-op; b, 1 month post-op vs. 1 week post-op and 3 months post-op; c, baseline vs. 1 day post-op; d, 1 month post-op vs. 1 day post-op, 1 week post-op respectively; e, 1 month post-op vs. baseline, 1 day post-op and 1 week post-op; f, 3 months vs. baseline, 1 day post-op and 1 week post-op. The data were analyzed by generalized estimating equations with Bonferroni corrections. Groups: A, high myopia; B, low myopia. RT, retinal thickness; SVD, superficial vascular density; DVD, deep vascular density.
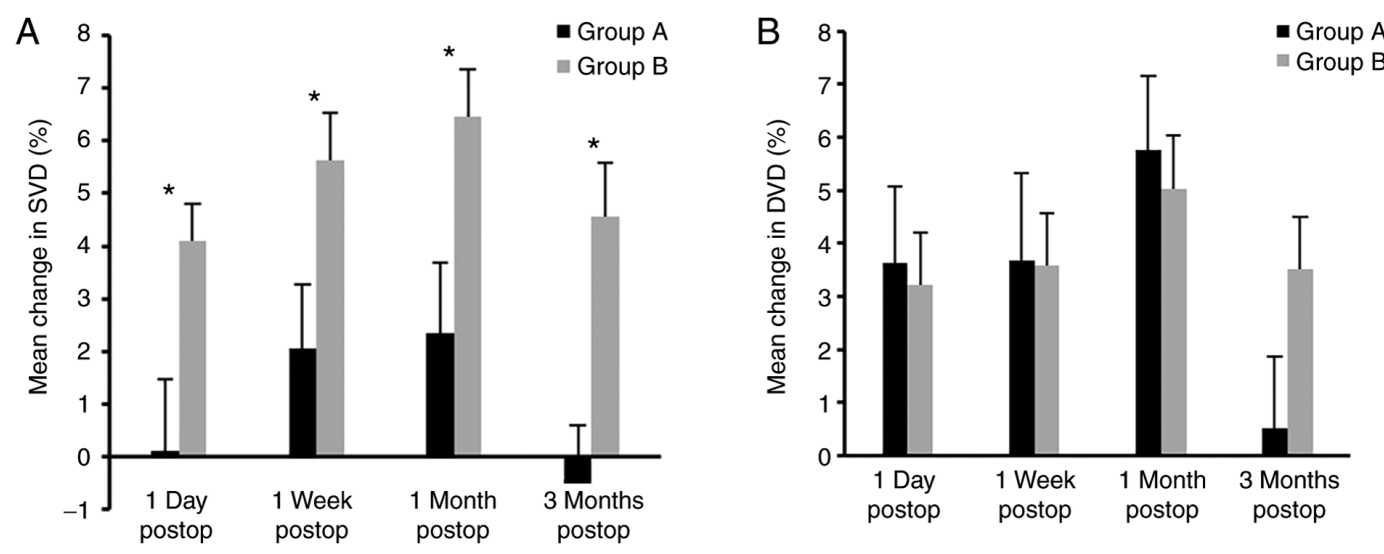

Figure 3. Mean change (\%) in SVD and DVD at four time-points after surgery in the two groups. (A) SVD and (B) DVD. *P<0.05. P-values were calculated by generalized estimating equations. Groups: A, high myopia; B, low myopia. SVD, superficial vascular density; DVD, deep vascular density.

between the two groups at 1 day, 1 week, 1 and 3 months post-operatively $(\mathrm{P}=0.009, \mathrm{P}=0.02, \mathrm{P}=0.012$ and $\mathrm{P}=0.001$, respectively; Fig. 3A and Table IV). Furthermore, the difference was mainly observed in the parafovea $(\mathrm{SH}, \mathrm{IH}, \mathrm{T}, \mathrm{S}, \mathrm{N}$ and I quadrants) but not in the fovea $(\mathrm{P}>0.05)$. However, the mean changes in DVD were not significantly different between the two groups at the four time-points after cataract surgery $(\mathrm{P}=0.808,0.962,0.695$ and 0.079 , respectively; Fig. 3B and Table IV).

Correlation between age, BCVA, AL, RT, IOP and SVD. Pearson's correlation test revealed that the negative correlations 

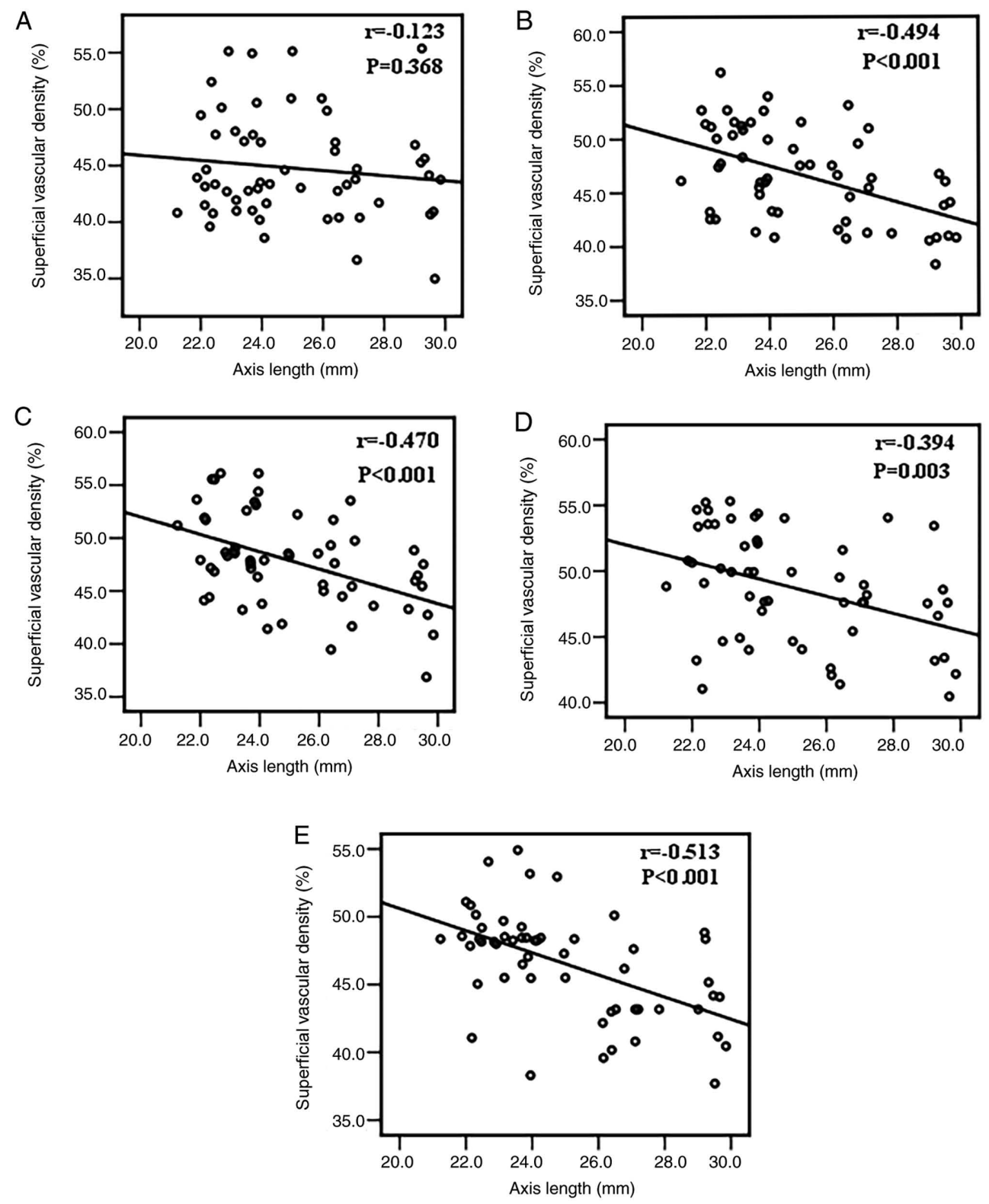

Figure 4. Correlation between axial length and superficial vascular density. (A) Baseline. (B) 1 day post-op. (C) 1 week post-op. (D) 1 month post-op. (E) 3 months post-op. The $r$ value is the correlation coefficient. P-values were calculated using Pearson's correlation.

between AL and SVD were more significant at 1 day, 1 week, and 1 and 3 months after surgery $(\mathrm{r}=-0.494, \mathrm{P}<0.001 ; \mathrm{r}=-0.470$, $\mathrm{P}<0.001 ; \mathrm{r}=-0.394, \mathrm{P}=0.003$; and $\mathrm{r}=-0.513, \mathrm{P}<0.001$, respectively) than those prior to surgery ( $\mathrm{r}=-0.123, \mathrm{P}=0.368$; Fig. 4). The superficial RT in the parafovea was positively correlated with the SVD after 1 day, 1 week and 1 month after surgery $(\mathrm{r}=0.298, \mathrm{P}=0.024 ; \mathrm{r}=0.261, \mathrm{P}=0.050$; and $\mathrm{r}=0.377, \mathrm{P}=0.010$, respectively; Fig. 5). Furthermore, the IOP was negatively correlated with the SVD at 1 month after surgery $(r=-0.355$, $\mathrm{P}=0.01$ ) while no association was determined at any other time-point $(\mathrm{P}>0.05)$ (data not shown). However, age and BCVA were not correlated with SVD ( $>0.05)$ (data not shown).

\section{Discussion}

Clinically, there have been certain reports of ischemic optic neuropathy occurring after cataract surgery and leading to a sudden decline in VA $(3,4)$. Another study suggested that the macular vascular system was essential to visual function (5). In patients with high myopia, there are certain structural changes, including retinal and choroidal thickness thinning, optic disc atrophy and thinning of RNFL thickness. In addition, the retinal blood flow and blood vessel diameter were indicated to be decreased in high-myopia patients with Doppler ultrasound (8). A number of these studies used Doppler and FFA to detect retinal perfusion. However, Doppler is not able to sensitively measure the macular and peripapillary microcirculation (5). Azizi et al (15) indicated that retinal vessel densitometry with Doppler may result in an erroneous elevation in the detection of cataracts due to cataract-induced light scatter. FFA is not able to differentiate the deep capillary from the radial peripapillary networks (16). In the present study, non-invasive OCTA was used with the SSADA algorithm for imaging to quantify the MVD with high resolution and 

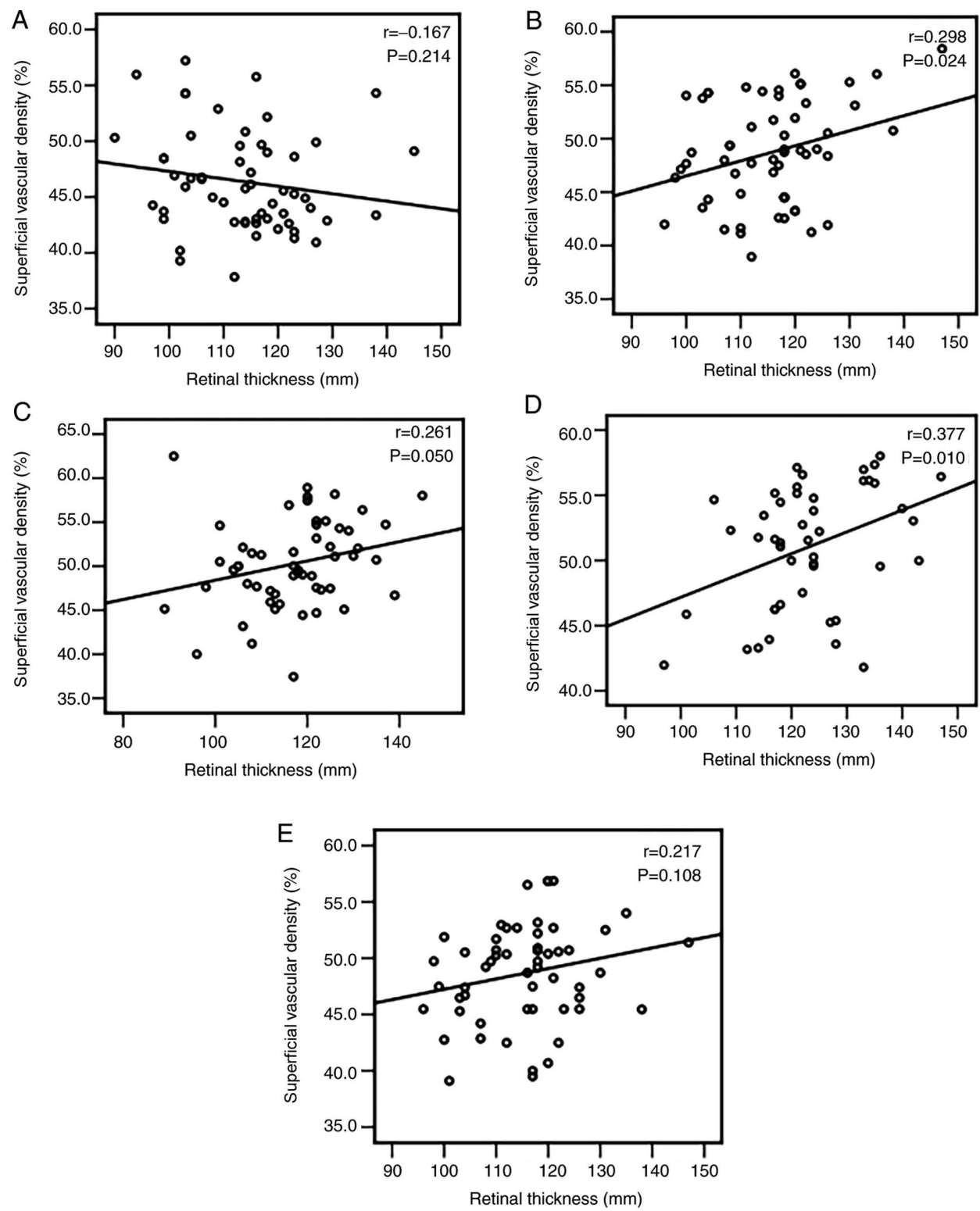

Figure 5. Correlation between superficial vascular density and superficial retinal thickness in the parafovea. (A) Baseline. (B) 1 day post-op. (C) 1 week post-op. (D) 1 month post-op. (E) 3 months post-op. The $\mathrm{r}$ value is the correlation coefficient. P-values were calculated by Pearson's correlation.

three-dimensional images. Good repeatability and reproducibility were reported for this method (5). OCTA is able to reveal more details about larger blood vessels and capillary networks in the superficial and deep retinal layers (17). These powerful features of OCTA have enormous implications for understanding tissue perfusion in retinal vascular diseases, including retinal venous occlusion, age-associated macular degeneration, idiopathic macular telangiectasia (18), diabetic retinopathy (19) and primary open-angle glaucoma (20). However, the use of OCTA for dynamic observation of retinal perfusion prior to and after cataract surgery in high myopia has been scarcely reported previously.

Cataract surgery has been widely used in the clinic and certain post-operative complications usually occur in the retina. For instance, one study indicated a subclinical increase in RT based on optical coherence tomography after phacoemulsification (21). In addition, pseudophakic cystoid macular edema leading to a decrease in VA were commonly observed after cataract surgery (22). A positive correlation between retinal perfusion and RT was reported in healthy subjects (23). Previous studies suggested different results for pulsatile ocular blood flow (POBF) changes detected using an OBF analyzer after cataract surgery. Hilton et al (24) suggested that the POBF was improved at 1 month following cataract surgery compared with that prior to surgery. However, Turk et al (25) determined that in normotensive eyes, cataract surgery did not affect ocular hemodynamics at 1 or 3 weeks post-surgery.

The present study suggested that MVD and RT in the high- and low-myopia groups changed after cataract surgery. A previous study reported changes in macular thickness and vessel density after uneventful cataract surgery. A 6-month follow-up study suggested that the average macular thickness exhibited the greatest increase at 1 month after cataract surgery and significantly decreased at 3 and 6 months after surgery (26). Zhao et al (27) determined that the MVD and thickness increased in patients with cataracts in the 3-month 
follow-up period after cataract surgery using OCTA, which was consistent with the results of the present study. Although the exact pathomechanism remains to be fully elucidated, the role of surgical trauma and the release of prostaglandins and blood retinal barrier disruption are likely causes (21). According to Zhao et al (27), the reason may be the decrease in IOP, post-operative inflammation or light exposure after cataract surgery. The present study also suggested that the IOP decreased at 3 months after surgery $(1.81-2.24 \mathrm{mmHg})$ compared with that of the baseline. Furthermore, the IOP exhibited a significantly positive correlation with SVD at the first post-operative month, which may further demonstrate the influence of the IOP on the vessels. However, it was indicated that the vessel density and RT fluctuated more obviously in patients with high myopia than in normal patients after surgery. This result indicated that the macular function in patients with high myopia was more susceptible to the influence of surgery. It was hypothesized that thinner retinal and choroidal thickness, decreased vessel density and thinner RNFL thickness in patients with high myopia (6), as well as decreased vessel diameter (7), may be attributed to this variation. Of note, both SVD and DVD exhibited a sudden significant decrease at 3 months after surgery in the high myopia group, which may indicate that the function of the retinal vessels of patients with high myopia was even worse than that of low myopia patients.

Although superficial vascular density increased after cataract surgery in both groups, the mean increase was lower in patients with high myopia than that in patients with low myopia. A possible reason may be the high IP used for maintaining IOP during surgery. One study suggested that a transient high IOP reduced the vessel diameters of the retina and optic disc in healthy rabbits and that the microcirculation gradually recovered to normal levels within $30 \mathrm{~min}$ after the high IOP was removed (28). The microcirculation perfusion was damaged when the IOP exceeded the autoregulatory capacity of the retinal vessels (29). In cataract surgery, the IP is maintained at a high level for a certain duration. IOP fluctuations during surgery caused by a high IP may affect retinal hemodynamics. Saygili et al (30) also demonstrated that increased IOP during cataract surgery decreased the flow velocity of the central retinal artery, which supplied the inner retinal layer with colorful Doppler and thereby affected the retinal perfusion. The present study indicated that the retinal vascular density was lower in the high myopia group than that in the low myopia group after surgery based on OCTA, which suggested that the retinal vascular perfusion was also lower in patients with high myopia than in patients with low myopia. A number of adverse factors in high myopia, including longer AL, reduced retinal and choroidal thickness, reduced RNFL thickness (6) and decreased vessel diameter (7), may affect the macular vascular system. The retinal vessels in patients with high myopia may be less able to tolerate the high IOP fluctuations during surgery that occur for a relatively long time. This eventually leads to insufficient self-regulation and inadequate perfusion of superficial retinal vessels after cataract surgery. In addition, the post-operative IOP of patients with high myopia was significantly higher than that of the low-myopia group, and low myopia may also be the reason for lower vascular density after the operation. Furthermore, the SVD of eyes with high myopia was even lower at 3 months compared with that at baseline. This result also indicated that patients with high myopia may exhibit lower retinal perfusion over a period of time. This suggests that it may be necessary to monitor retinal perfusion in patients with high myopia after surgery in the long term, which has not been previously reported and further research is required to support this notion.

Furthermore, the present study indicated a negative correlation between AL and SVD, which was consistent with previous studies. Fan et al (7) recruited participants under 40 years of age with different refractive statuses, including a control group $(\mathrm{AL}=23.28 \mathrm{~mm})$, a moderate group $(\mathrm{AL}=24.98 \mathrm{~mm})$ and a high-myopia group $(\mathrm{AL}=29.01 \mathrm{~mm})$, and determined by using OCTA that the vascular density decreased in the macular region with a longer AL but not in the disc region. Lam et al (31) demonstrated that the outer macular thickness decreased with a longer AL, while the central macular thickness increased with OCT in patients with myopia and pathologic myopia between 40 and 50 years of age. In addition, the present study indicated that the correlation exhibited a dynamic and changing tendency after cataract surgery, which had not been mentioned in previous studies. A reason for the changes in relevance may be that the post-operative inflammation induced by surgical trauma at different time-points affected retinal perfusion.

In addition, the present study determined that vessel density and RT remained positively correlated after surgery. The SVD had a significant positive correlation with the superficial RT but not with the full RT of the parafovea at an early stage after surgery, which was also demonstrated by other studies. For instance, using OCTA, Yu et al (23) demonstrated that the inner RT (from the ILM to the inner nuclear layer) was positively correlated with vascular density in both the macular and peripapillary areas in healthy subjects. Fan et al (7) indicated a positive correlation between MVD and GCL thickness, which was mainly supplied by superficial retinal vessels. Of note, the present study suggested that the correlation continued to change from mild to moderate after cataract surgery, which demonstrated that vascular density and RT may vary between different time-points. It was assumed that the thinning of the RT resulted in decreased oxygen demand and the blood microcirculation may consequently be reduced (9). However, it is possible that the vessel density changed prior to RT, which requires further study.

Mo et al (5) also indicated that the BCVA was positively correlated with the macular flow density among patients with pathological myopia. However, in the present study, no significant correlation was obtained between BCVA and MVD, neither prior to nor after surgery. Patients with high myopia without pathological myopia were enrolled in the present study and $83 \%$ of the patients with high myopia were classified as having peripapillary staphyloma. The majority of patients with high myopia had no macular atrophy, which may explain the absence of a correlation between BCVA and MVD in the present study. Patients with severe cataracts and macular atrophy were excluded from the present study because it was observed that these patients did not cooperate well during the examination and the data measured using OCTA were unreliable.

There are certain limitations to the present study. First, the number of patients included was relatively small and a 
future study with a larger sample size may provide further information about MVD variation after phacoemulsification. Furthermore, the density of the cataract may influence the accuracy of the measurements of vascular density and RT. Finally, additional types of high myopia should be included in further studies to examine the variation of retinal perfusion after cataract surgery in those patients.

In conclusion, superficial retinal perfusion was lower after phacoemulsification in patients with high myopia compared with that of patients with normal cataract, which exposed patients to the risk of experiencing complications that are caused by poor perfusion. Therefore, OCTA should be performed on post-operative patients with high myopia who are at high risk of ischemia.

\section{Acknowledgements}

The authors thank Mr. Chen Lei, ophthalmic technician at the Department of Ophthalmology of Shanghai 10th People's Hospital affiliated to Tongji University who helped make measurements for patients with OCTA.

\section{Funding}

This study was funded by the Shanghai Shenkang Clinical Research Cultivation Project (grant no. SHDC12019X30).

\section{Availability of data and materials}

The datasets used and/or analyzed during the current study are available from the corresponding author on reasonable request.

\section{Authors' contributions}

All authors contributed to the conception and design of the study. TL collected the patient information, analyzed the data and wrote the manuscript draft. AG was involved in drafting the manuscript and analysis and interpretation of the data. LF and JF participated in data collection and data analysis. ZJ performed data and statistical analysis. FL designed the experiments, analyzed and discussed the data, had an important role in interpreting the results and approved the final version of the manuscript. All authors read and approved the final manuscript.

\section{Ethics approval and consent to participate}

The subjects provided written informed consent and the study protocol was approved by the Clinical Research Ethical Committee of Shanghai 10th People's Hospital affiliated to Tongji University (Shanghai, China).

\section{Patient consent for publication}

Not applicable.

\section{Competing interests}

The authors declare that they have no competing interests.

\section{References}

1. Eom SY, Yim DH, Kim JH, Chae JB, Kim YD and Kim H: A pilot exome-wide association study of age-related cataract in Koreans. J Biomed Res 30: 186-190, 2016.

2. Mohammadi A, Khorasani N, Moloudi F and Ghasemi-Rad M: Evaluation of retrobulbar blood flow in patients with age-related cataract; color Doppler ultrasonographic findings. Clin Ophthalmol 5: 1521-1524, 2011.

3. Emeriewen K, Kadare S, Tsatsos M, Athanasiadis Y, MacGregor C and Rassam S: Non-arteritic anterior ischaemic optic neuropathy after uneventful cataract extraction. Neuroophthalmology 40: 225-228, 2016.

4. Al-Madani MV, Al-Raqqad NK, Al-Fgarra NA, Al-Thawaby AM and Jaafar AA: The risk of ischemic optic neuropathy post phacoemulsification cataract surgery. Pan Afr Med J 28: 53, 2017.

5. Mo J, Duan A, Chan S, Wang X and Wei W: Vascular flow density in pathological myopia: An optical coherence tomography angiography study. BMJ Open 7: e013571, 2017.

6. Ng DS, Cheung CY, Luk FO, Mohamed S, Brelen ME, Yam JC, Tsang CW and Lai TY: Advances of optical coherence tomography in myopia and pathologic myopia. Eye (Lond) 30: 901-916, 2016.

7. Fan H, Chen HY, Ma HJ, Chang Z, Yin HQ, Ng DS, Cheung CY, Hu S, Xiang X, Tang SB and Li SN: Reduced macular vascular density in myopic eyes. Chin Med J (Engl) 130: 445-451, 2017.

8. Benavente-Pérez A, Hosking SL, Logan NS and Broadway DC: Ocular blood flow measurements in healthy human myopic eyes. Graefes Arch Clin Exp Ophthalmol 248: 1587-1594, 2010.

9. Wang X, Kong X, Jiang C, Li M, Yu J and Sun X: Is the peripapillary retinal perfusion related to myopia in healthy eyes? A prospective comparative study. BMJ Open 6: e010791, 2016.

10. Nagiel A, Sadda SR and Sarraf D: A promising future for optical coherence tomography angiography. JAMA Ophthalmol 133: 629-630, 2015.

11. Jia Y, Bailey ST, Hwang TS, McClintic SM, Gao SS, Pennesi ME, Flaxel CJ, Lauer AK, Wilson DJ, Hornegger J, et al: Quantitative optical coherence tomography angiography of vascular abnormalities in the living human eye. Proc Natl Acad Sci USA 112: E2395-E2402, 2015.

12. Chylack LT Jr, Wolfe JK, Singer DM, Leske MC, Bullimore MA, Bailey IL, Friend J, McCarthy D and Wu SY: The lens opacities classification system III. The longitudinal study of cataract study group. Arch Ophthalmol 111: 831-836, 1993.

13. Curtin BJ: The posterior staphyloma of pathologic myopia. Trans Am Ophthalmol Soc 75: 67-86, 1977.

14. Friedel JE, DeHart WB, Foreman AM and Andrew ME: A Monte Carlo method for comparing generalized estimating equations to conventional statistical techniques for discounting data. J Exp Anal Behav 111: 207-224, 2019.

15. Azizi B, Wong T, Wan J, Singer S and Hudson C: The impact of cataract on the quantitative, non-invasive assessment of retinal blood flow. Acta Ophthalmol 90: e9-e12, 2012.

16. Spaide RF, Klancnik JM Jr and Cooney MJ: Retinal vascular layers imaged by fluorescein angiography and optical coherence tomography angiography. JAMA Ophthalmol 133: 45-50, 2015.

17. Mastropasqua R, Di Antonio L, Di Staso S, Agnifili L, Di Gregorio A, Ciancaglini M and Mastropasqua L: Optical Coherence tomography angiography in retinal vascular diseases and choroidal neovascularization. J Ophthalmol 2015: 343515, 2015.

18. Chalam KV and Sambhav K: Optical coherence tomography angiography in retinal diseases. J Ophthalmic Vis Res 11: 84-92, 2016.

19. Agemy SA, Scripsema NK, Shah CM, Chui T, Garcia PM, Lee JG, Gentile RC, Hsiao YS, Zhou Q, Ko T and Rosen RB: Retinal vascular perfusion density mapping using optical coherence tomography angiography in normals and diabetic retinopathy patients. Retina 35: 2353-2363, 2015.

20. Yarmohammadi A, Zangwill LM, Manalastas PIC, Fuller NJ, Diniz-Filho A, Saunders LJ, Suh MH, Hasenstab K and Weinreb RN: Peripapillary and macular vessel density in patients with primary open-angle glaucoma and unilateral visual field loss. Ophthalmology 125: 578-587, 2018.

21. Gharbiya M, Cruciani F, Cuozzo G, Parisi F, Russo P and Abdolrahimzadeh S: Macular thickness changes evaluated with spectral domain optical coherence tomography after uncomplicated phacoemulsification. Eye (Lond) 27: 605-611, 2013. 
22. Grzybowski A, Sikorski BL, Ascaso FJ and Huerva V: Pseudophakic cystoid macular edema: Update 2016. Clin Interv Aging 11: 1221-1229, 2016.

23. Yu J, Gu R, Zong Y, Xu H, Wang X, Sun X, Jiang C, Xie B, Jia Y and Huang D: Relationship between retinal perfusion and retinal thickness in healthy subjects: An optical coherence tomography angiography study. Invest Ophthalmol Vis Sci 57: OCT204-OCT210,2016.

24. Hilton EJ, Hosking SL, Gherghel D, Embleton S and Cunliffe IA: Beneficial effects of small-incision cataract surgery in patients demonstrating reduced ocular blood flow characteristics. Eye (Lond) 19: 670-675, 2005.

25. Turk A, Mollamehmetoglu S, Imamoglu HI, Kola M, Erdol H and Akyol N: Effects of phacoemulsification surgery on ocular hemodynamics. Int J Ophthalmol 6: 537-541, 2013.

26. Šiško K, Knez NK and Pahor D: Influence of cataract surgery on macular thickness: A 6-month follow-up. Wien Klin Wochenschr 127 (Suppl 5): S169-S174, 2015.
27. Zhao Z, Wen W, Jiang C and Lu Y: Changes in macular vasculature after uncomplicated phacoemulsification surgery: Optical coherence tomography angiography study. J Cataract Refract Surg 44: 453-458, 2018.

28. Zhao YY, Chang PJ, Yu F and Zhao YE: Retinal vessel diameter changes induced by transient high perfusion pressure. Int J Ophthalmol 7: 602-607, 2014.

29. Fortune B, Choe TE, Reynaud J,Hardin C, Cull GA, Burgoyne CF and Wang L: Deformation of the rodent optic nerve head and peripapillary structures during acute intraocular pressure elevation. Invest Ophthalmol Vis Sci 52: 6651-6661, 2011.

30. Saygili O, Mete A, Mete A, Gungor K, Bekir N and Bayram M: Does phacoemulsification under topical anesthesia affect retrobulbar blood flow? J Clin Ultrasound 40: 572-575, 2012.

31. Lam DS, Leung KS, Mohamed S, Chan WM, Palanivelu MS, Cheung CY, Li EY, Lai RY and Leung CK: Regional variations in the relationship between macular thickness measurements and myopia. Invest Ophthalmol Vis Sci 48: 376-382, 2007. 